\title{
APPROXIMATIONS OF STOCHASTIC INTEGRALS OF THE POISSON PROCESS
}

\author{
Gharib Musa Ibrahim Gharib \\ Mathematics Department, College of Science, Zarqa University, Jordan
}

ggharib@zu.edu.jo

"This research is funded by the Deanship of Research and Graduate Studies in Zarqa University/

\section{ABSTRACT} Jordan"

In this article the asymptotic behavior of the finite sums of the elements depending on approximations, being linear combinations of approximations by $\mathrm{I}$ to and Stratonovich, is investigated. The outcomes of the present work are generalizations of the corresponding theorems from

\section{Indexing terms/Keywords}

Stochastic integral; Poisson Process.

\section{Academic Discipline And Sub-Disciplines}

Applied Mathematics.

\section{SUBJECT CLASSIFICATION}

stochastic differential equations

\section{TYPE (METHOD/APPROACH)}

the convergence of the integral sums of Poisson

\section{INTRODUCTION}

For giving a mathematical meaning to the stochastic differential equations, in which the controlling process is a wiener process, the stochastic differentials (integrals) were defined. Initially stochastic integrals of Ito and Stratonovich were used as a base integrals, the first integral more often is operated by mathematics, and the second one is operated by natural scientists (in particular case, by physicists). Numerous generalizations of these integrals now are known : the $\theta$-stochastic integral, Ogawa stochastic integral etc. [2],[3],[4].

On the basis of generalized random processes algebra the space of generalized stochastic differentials was constructed in the article [1]. And the "symmetrical" subsets, which correspond to differentials by I to and Stratonovich at an associated level, were allocated in this space. In the work [5] the problem of approximation of a stochastic $\theta$-integral of the Brownian motion random process by the elements of generalized random processes algebra was investigated.

The second important class of exterior fluctuations is an appearance of the specific discrete events at the random moments of time in the mentioned space. The Poisson process is an appropriate model in this case. It is interesting to know that will turn out if refuse similarly the independence of the increments of Poisson process. In the work [6] the convergence of the integral sums of Poisson for the cases, where approximation is I to and Stratonovich approximation, was investigated. In this article the asymptotic behavior of the finite sums of the elements depending on approximations, being linear combinations of approximations by I to and Stratonovich, is investigated. The outcomes of the present work are generalizations of the corresponding theorems from [6].

\section{PAGE SIZE}

\section{Basic outcomes}

Let $\left(\Omega, A_{y} P\right)$ be full probability space; $\Pi(t, \omega), t \in T_{y} \omega \in \Omega$ be random Poisson process, which trajectories are continuous on the right; $\rho(t)_{3} t \in R$ be non-negative indefinitely differentiable function, which carrier from $[0,1]$ and $\int_{R} \rho(s) d s=1$, and $\rho_{n}^{1}(t)=n \rho(n t), \quad \rho_{n}^{2}(t)=\varphi(n) \rho(\varphi(n) t), \quad n \in N_{s}$ where $\varphi(n)$ is a monotonically nondecreasing sequence, $0<h_{n}<2 h_{n}<\cdots<m h_{n}=a$ be partition of the segment $T=[0, a]$. Then, for any ${ }^{t \in T}$ there are $\tau_{t} \in\left[0, h_{n}\right]$ and $m_{t} \in N_{s}$ that $t=\tau_{1}+m_{t} h_{n^{x}}$ 
As generalized Poisson process let us consider

$\Pi_{n}^{\theta}(t, \omega)=\theta \Pi_{n}^{1}(t, \omega)+(1-\theta) \Pi_{n}^{2}(t, \omega), \quad \theta \in[0,1], \quad$ where

$\Pi_{n}^{1}(t, \omega)=\int_{0}^{1 / n} \Pi\left(t+s_{y} \omega\right) \rho_{n}^{1}(s) d s, \quad \Pi_{n}^{2}\left(t_{p} \omega\right)=\int_{0}^{1 / s_{n}} \Pi\left(t+s_{y} \omega\right) \rho_{n}^{2}(s) d s$.

Theorem 1. Let $f \in c^{1}(R)_{n} f_{n}=f^{*} \rho_{n}^{1} n \longrightarrow \infty_{n}, h_{n} \longrightarrow 0, \frac{1}{\varphi(n)}=o\left(h_{n}\right)_{n} h_{n}=o\left(\frac{1}{n}\right)$.

\section{Then}

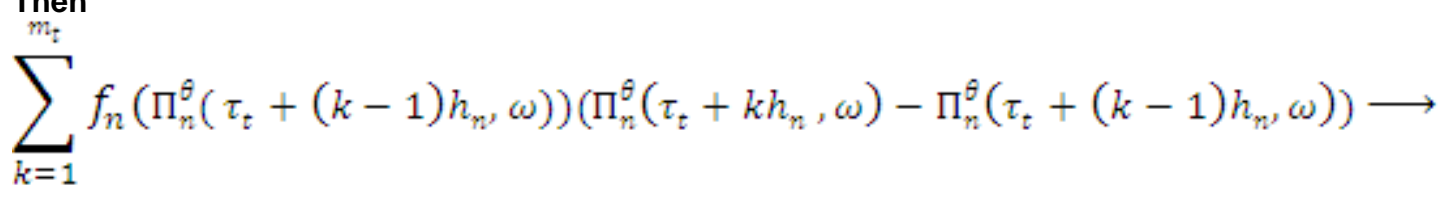

$\longrightarrow \int_{0}^{t}[g(\Pi(s-0, \omega)+\theta)-g(\Pi(s-0, \omega))] d \Pi(s, \omega)+(1-\theta) \int_{0}^{t} f(\Pi(s-0, \omega)+\theta) d \Pi(s, \omega)$

In the $L_{p}(T), p \geq 1$, for almost all $\omega \in \Omega$, where $g(x)=\int_{0}^{\infty} f(s) d s$.

The integral everywhere is understood in a sense of the Lebesgue - Stieltjes for almost all $\omega \in \Omega$.

$\sum_{k=1}^{\substack{\text { Then } \\ m n_{t}}} f_{n}\left(\Pi_{w 2}^{\theta}\left(\tau_{t}+k h_{m}, \omega\right)\right)\left(\Pi_{w}^{\theta}\left(\tau_{t}+k h_{w}, \omega\right)-\Pi_{w}^{\theta}\left(\tau_{t}+(k-1) h_{w}, \omega\right)\right) \longrightarrow$
$\longrightarrow \int_{0}^{t}[g(\Pi(s-0, \omega)+\theta)-g(\Pi(s-0, \omega))] d \Pi(s, \omega)+(1-\theta) \int_{0}^{t} f(\Pi(s, \omega)) d \Pi(s, \omega)$

Theorem 2. Let $f \in c^{1}(R), f_{n}=f^{*} \rho_{n}^{1}, n \longrightarrow \infty, h_{n} \longrightarrow 0, \frac{1}{\varphi(n)}=o\left(h_{n}\right), h_{n}=o\left(\frac{1}{n}\right)$.

In the $L_{p}(T), p \geq 1$, for almost all $\omega \in \Omega$.

As generalized Poisson process now let us consider

$\Pi_{n}^{\theta}(t, \omega)=\theta \Pi_{n}^{1}(t, \omega)+(1-\theta) \Pi_{n}^{2}(t, \omega), \quad \theta \in[0,1], \quad$ where

$\Pi_{n}^{1}(t, \omega)=\int_{0}^{1 / n} \Pi\left(t-s_{y} \omega\right) \rho_{n}^{1}(s) d s, \quad \Pi_{n}^{2}(t, \omega)=\int_{0}^{1 / n} \Pi\left(t-s_{y} \omega\right) \rho_{n}^{2}(s) d s$.

Theorem 3. Let $f \in c^{1}(R), f_{n}=f^{*} \rho_{n}^{1}, n \longrightarrow \infty, h_{n} \longrightarrow 0, \frac{1}{\varphi(n)}=o\left(h_{n}\right), h_{n}=o\left(\frac{1}{n}\right)$.

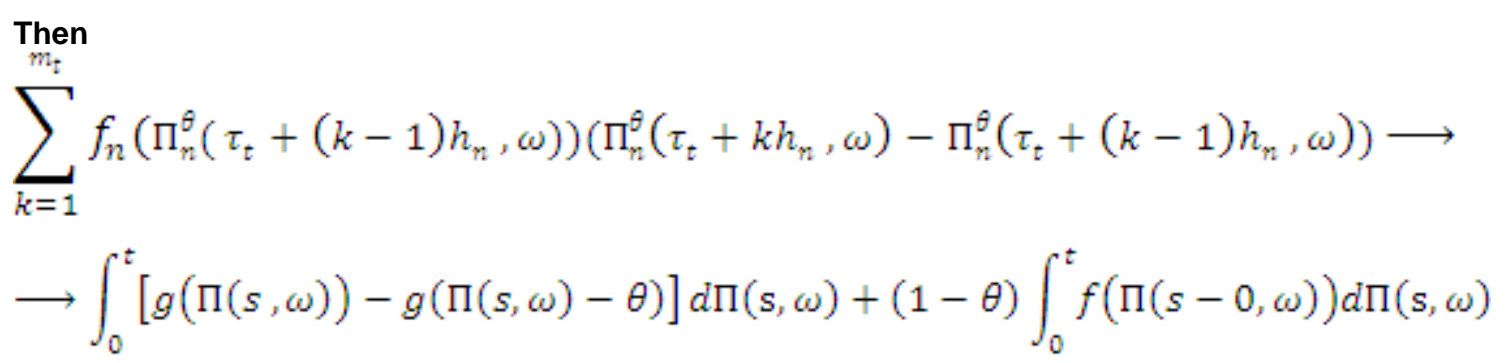

In the $L_{p}(T), p \geq 1$, for almost all $\omega \in \Omega$. 
Theorem 4. Let $f \in c^{1}(R), f_{n}=f^{*} \rho_{n}^{1}, n \longrightarrow \infty, h_{n} \longrightarrow 0, \frac{1}{\varphi(n)}=o\left(h_{n}\right), h_{n}=o\left(\frac{1}{n}\right)$.

$$
\begin{aligned}
& \sum_{k=1}^{\substack{\text { Then } \\
\theta n_{t}}} f_{n}\left(\Pi_{w}^{\theta}\left(\tau_{t}+k h_{n}, \omega\right)\right)\left(\Pi_{w}^{\theta}\left(\tau_{t}+k h_{w}, \omega\right)-\Pi_{w}^{\theta}\left(\tau_{t}+(k-1) h_{n}, \omega\right)\right) \longrightarrow \\
& \longrightarrow \int_{0}^{t}[g(\Pi(s, \omega))-g(\Pi(s, \omega)-\theta)] d \Pi(s, \omega)+(1-\theta) \int_{0}^{t} f(\Pi(s, \omega)-\theta) d \Pi(s, \omega)
\end{aligned}
$$

In the $L_{p}(T), p \geq 1$, for almost all $\omega \in \Omega$.

\section{Proof of the theorem 4.}

$$
\begin{aligned}
& \sum
\end{aligned}
$$

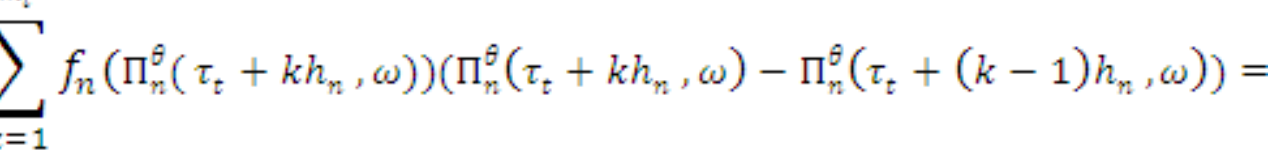$$
=\theta \sum_{k=1}^{m n_{t}} f_{n}\left(\Pi_{n}^{\theta}\left(\tau_{t}+k h_{n,}, \omega\right)\right)\left(\Pi_{w}^{1}\left(\tau_{t}+k h_{n}, \omega\right)-\Pi_{m}^{1}\left(\tau_{t}+(k-1) h_{n,} \omega\right)\right)+
$$$$
+(1-\theta) \sum_{k=1}^{m_{t}} f_{n}\left(\Pi_{n}^{\theta}\left(\tau_{t}+k h_{n}, \omega\right)\right)\left(\Pi_{n}^{2}\left(\tau_{t}+k h_{n}, \omega\right)-\Pi_{n}^{2}\left(\tau_{t}+(k-1) h_{n}, \omega\right)\right)=
$$$$
=\theta I_{n}^{1}(t, \omega)+(1-\theta) I_{n}^{2}(t, \omega)
$$

Let us show that $\theta I_{m}^{1} \longrightarrow \int_{0}^{t}[g(\Pi(s, \omega))-g(\Pi(s, \omega)-\theta)] d \Pi(\mathrm{s}, \omega)$

Let $g_{n}(x)=\int_{0}^{x} f_{n}(s) d s, x \in R_{y}$ then $g_{n} \longrightarrow g$ uniformly at any compact set from $R$.

$g_{n}\left(\Pi_{n}^{\theta}(t, \omega)\right)-g_{n}\left(\Pi_{n}^{\theta}(0, \omega)\right)=$

$=\sum_{k=1}^{m_{t}}\left(g_{n}\left(\Pi_{n}^{\theta}\left(\tau_{t}+k h_{n}, \omega\right)\right)-g_{n}\left(\Pi_{n}^{\theta}\left(\tau_{t}+(k-1) h_{n}, \omega\right)\right)\right)=$

$=\sum_{k=1}^{m_{t}}\left(g_{n}\left(\Pi_{n}^{\theta}\left(\tau_{t}+k h_{n}, \omega\right)\right)-g_{n}\left(\theta \Pi_{n}^{1}\left(\tau_{t}+(k-1) h_{n}, \omega\right)+(1-\theta) \Pi_{n}^{2}\left(\tau_{t}+k h_{n}, \omega\right)\right)\right)+$

$+\sum_{k=1}^{m_{t}}\left(g_{n}\left(\theta \Pi_{n}^{1}\left(\tau_{t}+(k-1) h_{n}, \omega\right)+(1-\theta) \Pi_{n}^{2}\left(\tau_{t}+k h_{n}, \omega\right)\right)-g_{n}\left(\Pi_{n}^{\theta}\left(\tau_{t}+(k-1) h_{n}, \omega\right)\right)\right)=$

$=J_{n}^{1}(t, \omega)+J_{n}^{2}(t, \omega)$.

Let us consider the first addend: $J_{n}^{1}(t, \omega)=$

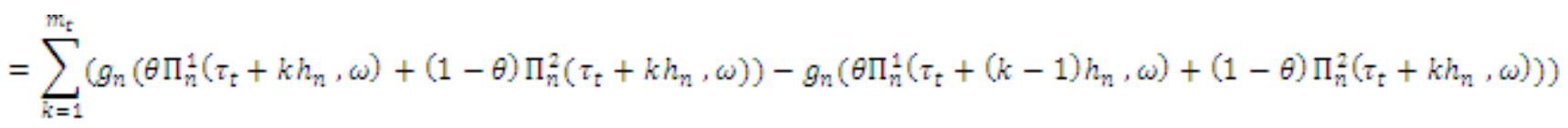




$$
\begin{aligned}
& =\theta \sum_{k=1}^{m m_{t}} f_{n}\left(\Pi_{w}^{\theta}\left(\tau_{t}+k h_{w}, \omega\right)\right)\left(\Pi_{w}^{1}\left(\tau_{t}+k h_{n}, \omega\right)-\Pi_{w}^{1}\left(\tau_{t}+(k-1) h_{w}, \omega\right)\right)+ \\
& +\frac{1}{2} \theta^{2} \sum_{k=1}^{m n_{t}} f_{n}\left(\overline{\theta_{n}}\right)\left(\Pi_{w}^{1}\left(\tau_{t}+k h_{w}, \omega\right)-\Pi_{w}^{1}\left(\tau_{t}+(k-1) h_{w}, \omega\right)\right)_{x}^{2}
\end{aligned}
$$

Where $\overline{\theta_{n}}$ is a point laying on a segment, connecting

$$
\begin{aligned}
& \theta \Pi_{n}^{1}\left(\tau_{t}+(k-1) h_{n}, \omega\right)+(1-\theta) \Pi_{n}^{2}\left(\tau_{t}+k h_{n}, \omega\right) \text { and } \\
& \theta \Pi_{n}^{1}\left(\tau_{t}+k h_{n}, \omega\right)+(1-\theta) \Pi_{n}^{2}\left(\tau_{t}+k h_{n}, \omega\right)
\end{aligned}
$$

From the lemma $1[7$, page 35$]$ follows that

$\frac{1}{2} \theta^{2} \sum_{k=1}^{m n_{t}} f_{n}\left(\overline{\theta_{n}}\right)\left(\Pi_{n}^{1}\left(\tau_{t}+k h_{n}, \omega\right)-\Pi_{w 2}^{1}\left(\tau_{t}+(k-1) h_{n}, \omega\right)\right)^{2} \rightarrow 0$ uniformly on ${ }^{\mathrm{t}}$ for almost all $\omega \in \Omega$

Let us designate the norm as $\|\cdot\|$ in the ${ }^{L}(T), p \geq 1$. We shall obtain that

$$
\begin{aligned}
& \left\|g_{n}\left(\theta \Pi_{n}^{1}(u, \omega)+(1-\theta) \Pi_{n}^{2}\left(u-\Delta_{y} \omega\right)\right)-g\left(\theta \Pi(u, \omega)+(1-\theta) \Pi\left(u-\Delta_{y} \omega\right)\right)\right\| \leq \\
& \leq\left\|g_{n}\left(\theta \Pi_{n}^{1}(u, \omega)+(1-\theta) \Pi_{n}^{2}\left(u-\Delta_{y} \omega\right)\right)-g\left(\theta \Pi_{n}^{1}(u, \omega)+(1-\theta) \Pi_{n}^{2}\left(u-\Delta_{y} \omega\right)\right)\right\|+ \\
& +\left\|g_{n}\left(\theta \Pi_{n}^{1}(u, \omega)+(1-\theta) \Pi_{n}^{1}\left(u-\Delta_{y} \omega\right)\right)-g\left(\theta \Pi(u, \omega)+(1-\theta) \Pi\left(u-\Delta_{y} \omega\right)\right)\right\| \leq \\
& \leq\left\|\int_{0}^{1 / n} g\left(\theta \Pi_{n}^{1}(u, \omega)+(1-\theta) \Pi_{n}^{2}\left(u-\Delta_{y} \omega\right)+s\right)-g\left(\theta \Pi_{n}^{1}(u, \omega)+(1-\theta) \Pi_{n}^{2}\left(u-\Delta_{y} \omega\right)\right) \rho_{n}(s) d s \cdot\right\|+ \\
& +\left\|g\left(\theta_{n}\right)\left\{\theta\left[\Pi_{n}^{1}(u, \omega)-\Pi(u, \omega)\right]+(1-\theta)\left[\Pi_{n}^{2}\left(u-\Delta_{y} \omega\right)-\Pi\left(u-\Delta_{y} \omega\right)\right]\right\}\right\| \leq \\
& \leq \frac{c(\omega)}{n}+c\left\|\Pi_{w}^{1}(u, \omega)-\Pi(u, \omega)\right\|+\left\|\Pi_{n}^{2}\left(u-\Delta_{y} \omega\right)-\Pi\left(u-\Delta_{y} \omega\right)\right\|_{y} \text { where } \Delta \geq 0, u-\Delta \geq 0 .
\end{aligned}
$$

The right part of the inequality aspires to zero at the $n \longrightarrow \infty$ for almost all $\omega \in \Omega$.

Thus, $g_{n}\left(\Pi_{n}^{\theta}(t, \omega)\right)-g_{n}\left(\Pi_{n}^{\theta}(0, \omega)\right) \longrightarrow g(\Pi(t, \omega))-g(\Pi(0, \omega))=$

$=\int_{0}^{t}\left[g\left(\Pi\left(s_{y} \omega\right)\right)-g(\Pi(s-0, \omega))\right] d \Pi(s, \omega)$ in the $L_{p}(T), p \geq 1$, for almost all $\omega \in \Omega$.

Now we shall prove that

$A_{n}(\omega)=\left\|j_{n}^{2}\left(t_{j} \omega\right)-\sum_{i=1}^{\Pi\left(t_{j} \omega\right)}\left[g\left(\theta \Pi\left(\mu_{i}-0, \omega\right)+(1-\theta) \Pi\left(\mu_{i}, \omega\right)\right)-g\left(\Pi\left(\mu_{i}-0, \omega\right)\right)\right]\right\| \longrightarrow 0$

for almost all $\omega \in \Omega$. 


$$
\begin{aligned}
A_{n}(\omega) \leq \| \sum_{k=1}^{m_{t}} & {\left[g_{n}\left(\theta \Pi_{n}^{1}\left(\tau_{t}+(k-1) h_{n}, \omega\right)+(1-\theta) \Pi_{n}^{2}\left(\tau_{t}+k h_{n}, \omega\right)\right)\right.} \\
& \left.-g_{n}\left(\theta \Pi_{n}^{1}\left(\tau_{t}+(k-1) h_{n}, \omega\right)+(1-\theta) \Pi_{n}^{2}\left(\tau_{t}+(k-1) h_{n}, \omega\right)\right)\right] \\
& -\sum_{k=1}^{m_{t}}\left[g_{n}\left(\theta \Pi_{n}^{1}\left(\tau_{t}+(k-1) h_{n}, \omega\right)+(1-\theta) \Pi_{n}^{2}\left(\tau_{t}+k h_{n}, \omega\right)\right)\right. \\
& \left.-g_{n}\left(\theta \Pi_{n}^{1}\left(\tau_{t}+(k-1) h_{n}, \omega\right)+(1-\theta) \Pi\left(\tau_{t}+(k-1) h_{n}, \omega\right)\right)\right] \|_{+}
\end{aligned}
$$$$
\begin{aligned}
+\| \sum_{k=1}^{m_{t}}\left[g_{n}\left(\theta \Pi_{n}^{1}\left(\tau_{t}+(k-1) h_{n}, \omega\right)+(1-\theta) \Pi\left(\tau_{t}+k h_{n}, \omega\right)\right)-g_{n}\left(\theta \Pi_{n}^{1}\left(\tau_{t}+(k-1) h_{n}, \omega\right)\right.\right. \\
\left.\left.+(1-\theta) \Pi\left(\tau_{t}+(k-1) h_{n}, \omega\right)\right)\right] \\
\quad-\sum_{i=1}^{\Pi\left(t_{0} \omega\right)}\left[g\left(\theta \Pi\left(\mu_{i}-0, \omega\right)+(1-\theta) \Pi\left(\mu_{i}-0, \omega\right)\right)-g\left(\Pi\left(\mu_{i}-0, \omega\right)\right)\right] \|=
\end{aligned}
$$$$
=A_{n}^{1}(\omega)+A_{n}^{2}(\omega) \text {. }
$$

Let us note, that $\Pi_{n}^{2}\left({ }^{*} \omega\right)$ differs from $\Pi\left({ }^{*} \omega\right)$ only at points $\tau_{t}+j h_{n,} 1 \leq j \leq m_{t}$, for which performs $\tau_{t}+j h_{n} \in\left(\mu_{i}, \mu_{i}+\frac{1}{\varphi(n)}\right), 1 \leq j \leq m_{t}, 1 \leq i \leq \Pi(t, \omega)$. ${ }_{\text {the set }} B_{n_{0}} t \in T_{y}$ for which $\tau_{t}+j h_{n}$ hit in the specified intervals, has a measure can be estimated above by $C(\omega) \frac{1}{\varphi(n) h_{n}}$. Under the investigation of $A_{n}^{1}(\omega)$ we shall use the previous relations:

$$
\begin{aligned}
& A_{n}^{1}(\omega)=\int_{E_{n}}\left\{\sum _ { k = 1 } ^ { m _ { t } } \left[g_{n}\left(\theta \Pi_{n}^{1}\left(\tau_{t}+(k-1) h_{n}, \omega\right)+(1-\theta) \Pi_{n}^{2}\left(\tau_{t}+k h_{n}, \omega\right)\right)\right.\right. \\
&\left.-g_{n}\left(\theta \Pi_{n}^{1}\left(\tau_{t}+(k-1) h_{n}, \omega\right)+(1-\theta) \Pi_{n}^{2}\left(\tau_{t}+(k-1) h_{n}, \omega\right)\right)\right] \\
&-\sum_{k=1}^{m_{t}}\left[g_{n}\left(\theta \Pi_{n}^{1}\left(\tau_{t}+(k-1) h_{n}, \omega\right)+(1-\theta) \Pi\left(\tau_{t}+k h_{n}, \omega\right)\right)\right. \\
&\left.\left.-g_{n}\left(\theta \Pi_{n}^{1}\left(\tau_{t}+(k-1) h_{n}, \omega\right)+(1-\theta) \Pi\left(\tau_{t}+(k-1) h_{n}, \omega\right)\right)\right]\right\} d t \leq c(\omega) \frac{1}{\varphi(n) h_{n}}
\end{aligned}
$$

Let us consider now $A_{n}^{2}(\omega) x$ 


$$
\begin{aligned}
& A_{n}^{2}(\omega) \leq \| \sum_{k=1}^{m_{t}}\left\{\left[g_{n}\left(\theta \Pi_{n}^{1}\left(\tau_{t}+(k-1) h_{n}, \omega\right)+(1-\theta) \Pi\left(\tau_{t}+k h_{n}, \omega\right)\right)\right.\right. \\
&\left.\quad-g_{n}\left(\theta \Pi_{n}^{1}\left(\tau_{t}+(k-1) h_{n}, \omega\right)+(1-\theta) \Pi\left(\tau_{t}+(k-1) h_{n}, \omega\right)\right)\right] \\
&-\left[g_{n}\left(\theta \Pi_{n}^{1}\left(\tau_{t}+(k-1) h_{n}, \omega\right)+(1-\theta) \Pi\left(\tau_{t}+(k-1) h_{n}, \omega\right)\right)\right. \\
&\left.-g_{n}\left(\theta \Pi_{n}^{1}\left(\tau_{t}+(k-1) h_{n}, \omega\right)+(1-\theta) \Pi\left(\tau_{t}+(k-1) h_{n}, \omega\right)\right)\right] \|_{+}
\end{aligned}
$$$$
\begin{aligned}
+\| \sum_{k=1}^{m_{t}}\left\{\left[g\left(\theta \Pi_{n}^{1}\left(\tau_{t}+(k-1) h_{n}, \omega\right)+(1-\theta) \Pi\left(\tau_{t}+k h_{n,}, \omega\right)\right)-g\left(\theta \Pi_{n}^{1}\left(\tau_{t}+(k-1) h_{n}, \omega\right)\right.\right.\right. \\
\left.\left.+(1-\theta) \Pi\left(\tau_{t}+(k-1) h_{n, s} \omega\right)\right)\right] \\
\quad-\left[g\left(\theta \Pi\left(\tau_{t}+(k-1) h_{n,} \omega\right)+(1-\theta) \Pi\left(\tau_{t}+(k-1) h_{n,} \omega\right)\right)\right. \\
\\
\left.\quad-g\left(\Pi\left(\tau_{t}+(k-1) h_{n}, \omega\right)\right)\right] \|+
\end{aligned}
$$$$
+\| \sum_{k=1}^{m_{t}}\left\{\left[g\left(\theta \Pi\left(\tau_{t}+(k-1) h_{n}, \omega\right)+(1-\theta) \Pi\left(\tau_{t}+k h_{n}, \omega\right)\right)-g\left(\Pi\left(\tau_{t}+(k-1) h_{n}, \omega\right)\right)\right]\right.
$$$$
-\sum_{i=1}^{\Pi\left(t_{0} \omega\right)}\left[g\left(\theta \Pi\left(\mu_{i}-0, \omega\right)+(1-\theta) \Pi\left(\mu_{i}, \omega\right)\right)-g\left(\Pi\left(\mu_{i}-0, \omega\right)\right)\right] \|=
$$$$
=A_{n}^{21}(\omega)+A_{n}^{22}(\omega)+A_{n}^{23}(\omega)
$$$$
A_{n}^{21}(\omega)=\| \sum_{i=1}^{n(t, \omega)}\left\{\left[g_{n}\left(\theta \Pi_{n}^{1}\left(\tau_{t}+\left(k_{i}-1\right) h_{n}, \omega\right)+(1-\theta) \Pi\left(\tau_{t}+k_{i} h_{n}, \omega\right)\right)-g_{n}\left(\theta \Pi _ { n } ^ { 1 } \left(\tau_{t}\right.\right.\right.\right.
$$$$
\left.\left.\left.+\left(k_{\tilde{i}}-1\right) h_{n}, \omega\right)+(1-\theta) \Pi\left(\tau_{t}+\left(k_{\tilde{i}}-1\right) h_{n}, \omega\right)\right)\right]
$$$$
-\left[g\left(\theta \Pi_{n}^{1}\left(\tau_{t}+\left(k_{i}-1\right) h_{n}, \omega\right)+(1-\theta) \Pi\left(\tau_{t}+k_{i} h_{n}, \omega\right)\right)\right.
$$$$
\left.\left.-g\left(\theta \Pi_{n}^{1}\left(\tau_{i}+\left(k_{i}-1\right) h_{n}, \omega\right)+(1-\theta) \Pi\left(\tau_{t}+\left(k_{i}-1\right) h_{n}, \omega\right)\right)\right]\right\} \| \longrightarrow 0
$$

For almost all $\omega \in \Omega{ }$ where ${ }^{\tau_{i}}+\left(k_{i}-1\right) h_{n}<\mu_{i} \leq \tau_{i}+k_{i} h_{n}$.

After implementation of the theorem by Lagrange: 


$$
\begin{aligned}
& A_{n}^{22}(\omega)=\| \sum_{i=1}^{n(t, \omega)}\left\{\left[g\left(\theta \Pi_{n}^{1}\left(\tau_{i}+\left(k_{i}-1\right) h_{n,}, \omega\right)+(1-\theta) \Pi\left(\tau_{i}+k_{i} h_{n,}, \omega\right)\right)-g\left(\theta \Pi _ { n } ^ { 1 } \left(\tau_{t}\right.\right.\right.\right. \\
& \left.\left.\left.+\left(k_{\tilde{i}}-1\right) h_{n}, \omega\right)+(1-\theta) \Pi\left(\tau_{t}+\left(k_{\tilde{i}}-1\right) h_{n}, \omega\right)\right)\right] \\
& -\left[g\left(\theta \Pi\left(\tau_{t}+\left(k_{i}-1\right) h_{n}, \omega\right)+(1-\theta) \Pi\left(\tau_{t}+k_{i} h_{n}, \omega\right)\right)\right. \\
& \left.\left.-g\left(\Pi\left(\tau_{t}+\left(k_{i}-1\right) h_{n}, \omega\right)\right)\right]\right\} \| \leq
\end{aligned}
$$$$
\leq\left\|\sum_{i=1}^{\Pi\left(t_{0} \omega\right)} \theta C\left[\Pi_{n}^{1}\left(\tau_{t}+\left(k_{i}-1\right) h_{n}, \omega\right)+\Pi\left(\tau_{t}+\left(k_{i}-1\right) h_{n}, \omega\right)\right]\right\| \longrightarrow 0
$$

for almost all $\omega \in \Omega$.

$$
\begin{aligned}
& A_{n}^{23}(\omega)=\| \sum_{i=1}^{\Pi\left(t_{i} \omega\right)}\left\{\left[g\left(\theta \Pi\left(\tau_{i}+\left(k_{i}-1\right) h_{n}, \omega\right)+(1-\theta) \Pi\left(\tau_{i}+k_{i} h_{n}, \omega\right)\right)-g\left(\Pi \left(\tau_{t}\right.\right.\right.\right. \\
&\left.\left.\left.\left.+\left(k_{i}-1\right) h_{n}, \omega\right)\right)\right]-\left[g\left(\theta \Pi\left(\mu_{i}-0, \omega\right)+(1-\theta) \Pi\left(\mu_{i}, \omega\right)\right)-g\left(\Pi\left(\mu_{i}-0, \omega\right)\right)\right]\right\} \| \\
& \rightarrow 0
\end{aligned}
$$

As a result for almost all $\omega \in \Omega$.

$\theta I_{n}^{1} \stackrel{L_{\mathrm{p}}(\tau)}{\longrightarrow} \int_{0}^{t}(g(\Pi(\mathrm{s}, \omega))-g(\Pi(\mathrm{s}-0, \omega)) d \Pi(\mathrm{s}, \omega)-$

$-\sum_{i=1}^{\Pi(s, \omega)}\left[g\left(\theta \Pi\left(\mu_{i}-0, \omega\right)+(1-\theta) \Pi\left(\mu_{i}, \omega\right)\right)-g\left(\Pi\left(\mu_{i}-0, \omega\right)\right)\right]=$

$=\int_{0}^{t}[g(\Pi(s, \omega))-g(\Pi(s, \omega)-\theta) d \Pi(s, \omega)$

at realization of the theorem conditions.

Now let us consider $I_{m}^{2}(t, \omega)$.

Let us show that

$I_{n}^{2}(t, \omega) \longrightarrow \int_{0}^{t} f(\Pi(\mathrm{s}, \omega)-\theta) d \Pi(\mathrm{s}, \omega)$ in the $L_{p}(T), p \geq 1_{p}$ for almost all $\omega \in \Omega$.

$\left\|\sum_{k=1}^{m_{t}} f_{n}\left(\Pi_{n}^{\theta}\left(\tau_{t}+k h_{n}, \omega\right)\right)\left(\Pi_{n}^{2}\left(\tau_{t}+k h_{n}, \omega\right)-\Pi_{n}^{2}\left(\tau_{t}+(k-1) h_{n}, \omega\right)\right)-\int_{0}^{t} f(\Pi(s, \omega)-\theta) d \Pi(s, \omega)\right\| \leq$

$\leq\left\|\sum_{k=1}^{m_{t}}\left(f_{n}\left(\Pi_{n}^{\theta}\left(\tau_{t}+k h_{n}, \omega\right)\right)-f\left(\Pi_{n}^{\theta}\left(\tau_{t}+k h_{n}, \omega\right)\right)\right)\left(\Pi_{n}^{2}\left(\tau_{t}+k h_{n}, \omega\right)-\Pi_{n}^{2}\left(\tau_{t}+(k-1) h_{n}, \omega\right)\right)\right\|+$ 


$$
\begin{gathered}
+\| \sum_{k=1}^{m_{t}}\left[f \left(\theta \Pi_{n}^{1}\left(\tau_{t}+k h_{n}, \omega\right)+(1-\theta)\left(\Pi_{n}^{2}\left(\tau_{t}+k h_{n}, \omega\right)\right)\right.\right. \\
\left.-f\left(\theta \Pi_{n}^{1}\left(\tau_{t}+k h_{n,} \omega\right)+(1-\theta) \Pi\left(\tau_{t}+k h_{n}, \omega\right)\right)\right]\left(\Pi_{n}^{2}\left(\tau_{t}+k h_{n}, \omega\right)\right) \\
-\Pi_{n}^{2}\left(\tau_{t}+(k-1) h_{n}, \omega\right) \|+
\end{gathered}
$$$$
+\| \sum_{k=1}^{m_{t}}\left[f \left(\theta \Pi_{n}^{1}\left(\tau_{t}+k h_{n,} \omega\right)+(1-\theta)\left(\Pi\left(\tau_{t}+k h_{n,} \omega\right)\right)-f\left(\Pi \left(\tau_{t}\right.\right.\right.\right.
$$$$
\left.\left.\left.+k h_{n,}, \omega\right)\right)\right]\left(\Pi_{n}^{2}\left(\tau_{t}+k h_{n}, \omega\right)-\Pi_{n}^{2}\left(\tau_{t}+(k-1) h_{n}, \omega\right)\right)-\sum_{i=1}^{\pi\left(t_{i} \omega\right)}[f(i-\theta)-f(i)] \|
$$$$
+
$$$$
\left.+\| \sum_{k=1}^{m_{t}} f\left(\Pi\left(\tau_{t}+k h_{n}, \omega\right)\right) \Pi_{n}^{2}\left(\tau_{t}+k h_{n}, \omega\right)-\Pi\left(\tau_{t}+k h_{n}, \omega\right)\right) \|+
$$$$
\left.+\| \sum_{k=1}^{m_{t}} f\left(\Pi\left(\tau_{t}+k h_{n}, \omega\right)\right) \Pi_{n}^{2}\left(\tau_{t}+(k-1) h_{n}, \omega\right)-\Pi\left(\tau_{t}+(k-1) h_{n}, \omega\right)\right) \|+
$$

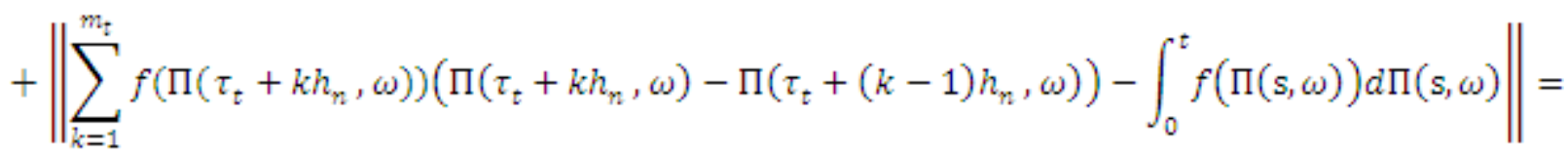$$
=I_{n}^{21}\left(t_{j} \omega\right)+I_{n}^{22}\left(t_{m} \omega\right)+I_{n}^{24}\left(t_{p} \omega\right)+I_{n}^{25}\left(t_{j} \omega\right)+I_{n}^{26}\left(t_{\nu} \omega\right) \text {. }
$$

$I_{n}^{26}(t, \omega) \longrightarrow 0$ by the definition.

Using the representation $f_{n}$, we shall obtain that $I_{n}^{21}\left(t_{p} \omega\right) \longrightarrow 0$.

$$
\begin{aligned}
& I_{n}^{22}(t, \omega)=\| \sum_{k=1}^{m_{t}}\left[f\left(\theta \Pi_{n}^{1}\left(\tau_{t}+k h_{n}, \omega\right)+(1-\theta) \Pi_{n}^{2}\left(\tau_{t}+k h_{n}, \omega\right)\right)-f\left(\theta \Pi_{n}^{1}\left(\tau_{t}+k h_{n}, \omega\right)\right.\right. \\
& \left.\left.+(1-\theta) \Pi\left(\tau_{t}+k h_{n}, \omega\right)\right)\right]\left(\Pi_{n}^{2}\left(\tau_{t}+k h_{n}, \omega\right)-\Pi_{n}^{2}\left(\tau_{t}+(k-1) h_{n}, \omega\right)\right) \| \leq
\end{aligned}
$$

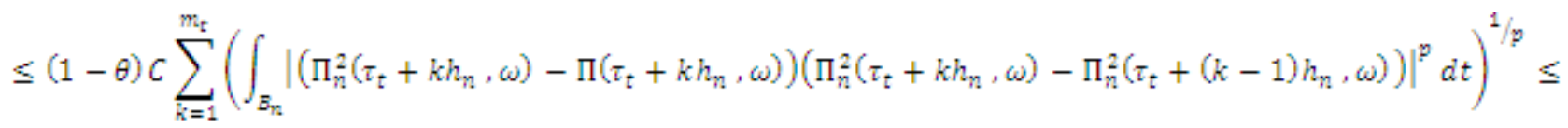

$$
\begin{aligned}
& \leq \frac{c}{\varphi(n) h_{n 2}} \longrightarrow 0 \text { at the realization of the theorem conditions. }
\end{aligned}
$$




$$
\begin{aligned}
& I_{n}^{23}(t, \omega)=\| \sum_{k=1}^{m_{t}}\left[f \left(\theta \Pi_{n}^{1}\left(\tau_{t}+k h_{n}, \omega\right)+(1-\theta)\left(\Pi\left(\tau_{t}+k h_{n}, \omega\right)\right)-f\left(\Pi \left(\tau_{t}\right.\right.\right.\right. \\
& \left.\left.\left.+k h_{n}, \omega\right)\right)\right]\left(\Pi_{n}^{2}\left(\tau_{t}+k h_{n}, \omega\right)-\Pi_{n}^{2}\left(\tau_{t}+(k-1) h_{n}, \omega\right)\right)-\sum_{i=1}^{\Pi\left(t_{t} \omega\right)}[f(i-\theta)-f(i)] \|= \\
& =\left(\int_{B_{n}} \mid \sum_{k=1}^{m m_{t}}\left[f \left(\theta \Pi_{n=1}^{1}\left(\tau_{t}+k h_{n 1}, \omega\right)+(1-\theta)\left(\Pi\left(\tau_{t}+k h_{n 2}, \omega\right)\right)-f\left(\Pi \left(\tau_{t}\right.\right.\right.\right.\right.
\end{aligned}
$$

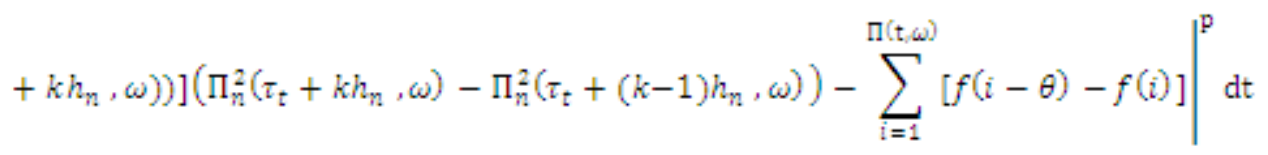

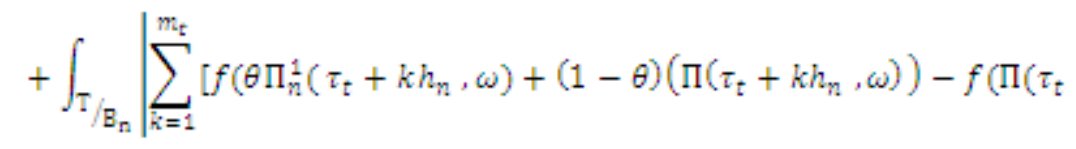

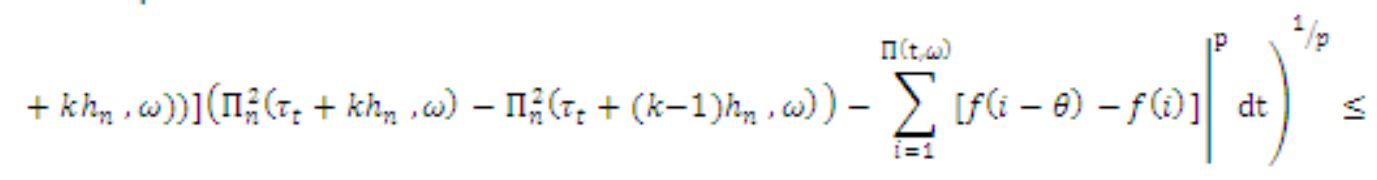

$$
\begin{aligned}
& \leq\left(\frac{c}{\varphi(n) h_{n}}+\int_{\mathrm{T}_{\mathrm{B}_{\mathrm{n}}}}\left|\sum_{\mathrm{i}=1}^{\mathrm{n}(\mathrm{t} \omega)}\left[f\left(\theta \Pi_{n}^{1}\left(\tau_{t}+k_{i} h_{n}, \omega\right)+(1-\theta) \Pi\left(\tau_{t}+k_{i} h_{n}, \omega\right)\right)-f(i-\theta)\right]\right|^{\mathrm{p}} \mathrm{dt}\right)^{1 / p} \leq \\
& \leq\left(\frac{C}{\varphi(n) h_{n}}+C \int_{T_{B_{n}}}\left|\sum_{i=1}^{\Pi(\mathrm{t} \omega)} \theta \int_{0}^{1 / h_{n}}\left(\Pi\left(\tau_{t}+k_{i} h_{n}-s, \omega\right)-\Pi\left(\tau_{t}+\left(k_{i}-1\right) h_{n}-s, \omega\right)\right) P_{n}(s) d s\right|^{p} d t\right)^{1 / p} \leq \\
& \leq\left(\frac{c}{\varphi(n) h_{n}}+c\left(n h_{n}\right)^{p}\right)^{1 / p} \longrightarrow 0 \\
& I_{n}^{24}\left(t_{,} \omega\right)=\left\|\sum_{k=1}^{m m_{t}} f\left(\Pi\left(\tau_{t}+k h_{n}, \omega\right)\right)\left(\Pi_{n}^{2}\left(\tau_{t}+k h_{n,}, \omega\right)-\Pi\left(\tau_{t}+k h_{n}, \omega\right)\right)\right\|= \\
& =\left(\int_{B_{n}}\left|\sum_{k=1}^{m_{t}} f\left(\Pi\left(\tau_{t}+k h_{n}, \omega\right)\right)\left(\Pi_{n}^{2}\left(\tau_{t}+k h_{n}, \omega\right)-\Pi\left(\tau_{t}+k h_{n}, \omega\right)\right)\right|^{p} d t\right)^{1 / p} \leq \frac{C}{\varphi(n) h_{n}} \longrightarrow 0 .
\end{aligned}
$$

Similarly, $I_{n}^{25}(t, \omega) \longrightarrow 0$.

Thus validity of the theorem is established.

Note: the theorems 1,2 and 3 are proved similarly. 


\section{REFERENCES}

1. Lazakovich, N.V. 1994 Stochastic Differentials in the Field of Generalized Random Processes Algebra.

2. Pugachev V.S., Sinitsyn I.N. 1987.Stochastic Differential Systems. Analysis and Filtering-Chichester.

3. Ogawa.B.S. 1970,On Riemann Definition of the Stochastic Integral

4. Lazakovich N.V., lablonsky O.L. Stochastic.1998. $\theta$ - Differentials in the Field of Generalized Random Processes Algebra.

5. Lazakovich N.V., Stashoulenok S.P. 1995.Approximation of Stochastic Integral of the Poisson Random Processes by the Generalized Processes Algebra Elements.

6. Lazakovich N.V., Stashoulenok S.P.1997. Approximation of the Integral and Equations of the Poisson Random Processes by the Meaning of Ito and Stratonovich.

7. Chung,K.L. Williams.R.J.1983. Introduction to Stochastic Integral. Progress in Probability and Statistics.

8. Lazakovich N.V., Lesnevsky V.E., Stashoulenok S.P.1998. $\theta$-Approximation of the Poisson Process. 